\title{
Deviations in Preoperative Fasting Guidelines in Children
}

\section{Kralik $S^{1}$, Kerovec Soric ${ }^{1}$, Kralik $M^{2}$ \\ ${ }^{1}$ Children's Hospital Zagreb, ${ }^{2}$ Clinical Hospital Center Zagreb, Croatia}

Background

It is routine clinical practice to follow the American Society of Anaesthesiologists (ASA) and European Society of Anaesthesiology (ESA) fasting guidelines before anaesthesia or sedation in children. In order to reduce the risk of regurgitation and pulmonary aspiration, these guidelines recommend abstaining from clear fluids for up to 2 hours, breast milk for up to 4 hours, and milk formulas and solids for up to 6 hours before anaesthesia or sedation.
Materials and Methods

Our prospective, observational study included 617 children undergoing anaesthesia or sedation from June 1 to December 12015 . During the preanaesthetic visit parents were informed about fasting guidelines and given written recommendations. Patient data and fasting times were recorded from the medical records immediately before anaesthesia or sedation. After the procedure, the fasting times were rechecked with a nurse or the parents.

\section{Results and Discussion}

The children were 10 days to 20 years old (median six years, mean \pm SD $6.87 \pm 4.98$ years), with body weight ranging from three to $135 \mathrm{~kg}$ (median $21 \mathrm{~kg}$, mean \pm SD $28.43 \pm 20.15 \mathrm{~kg}$ ). Preoperative fasting times ranged from two to 28 hours (median 11 hours, mean \pm SD $10.26 \pm 4.51$ hours) and the median deviation from the recommended preoperative fasting time was six hours (range -2 to 17 hours, mean \pm SD $5.86 \pm 3.61$ hours). Both age and body weight were positively correlated with both fasting duration (Figure 1) and deviation from the recommendations (Figure 2)( $<<0.01$ for all Pearson's and Spearman's correlation coefficients ranging from 0.134-0,248).
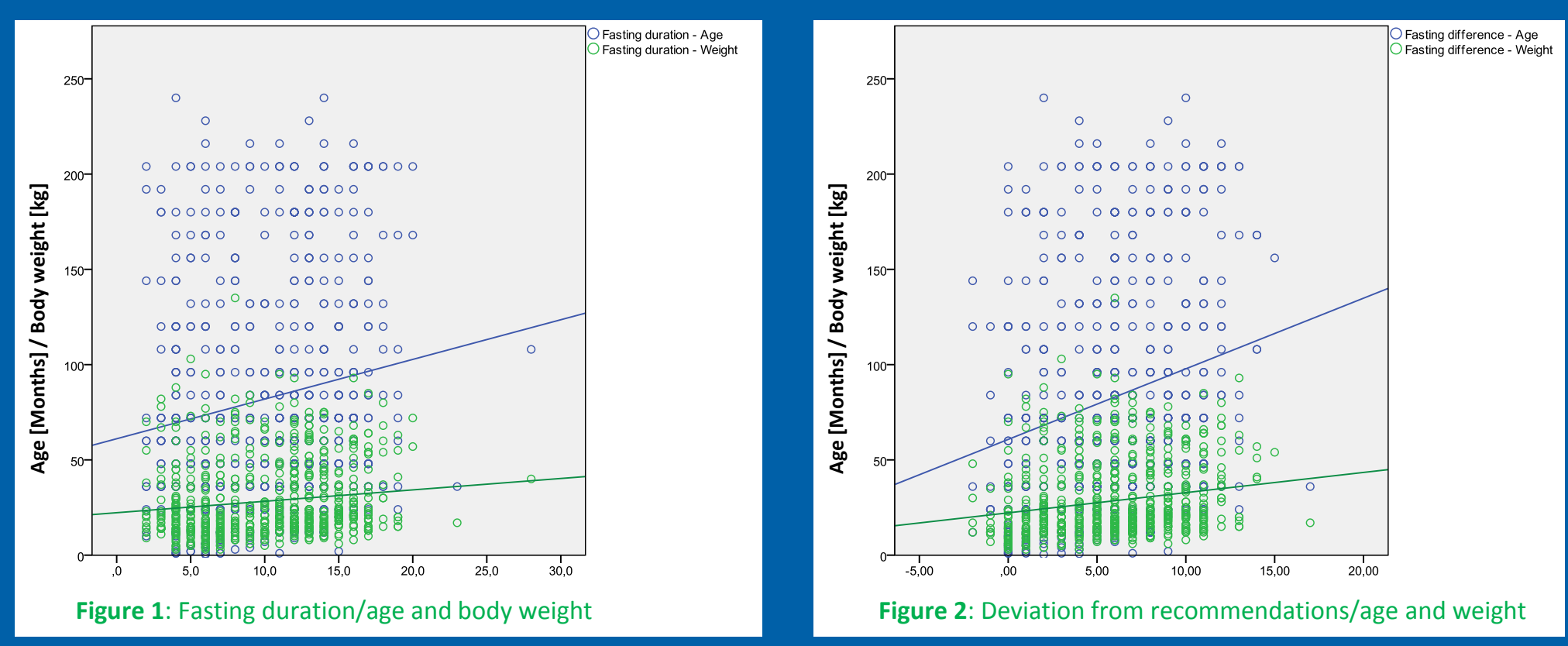

There were significant differences between median fasting times and deviations from the recommendations between different hospital departments, with pulmonology and intensive care units having the shortest median fasting time and the smallest median deviation, while urology and gastroenterology were on the opposite side of the spectrum (Kruskal-Wallis, both P<0.001). The same was true for the ASA groups: ASA III children had the smallest and ASA I children had the largest median values for both fasting time and deviation from the recommendations (Figure 3)(Kruskal-Wallis, both P<0.001). As expected, the median fasting time as well as the median deviation from the recommendations were significantly lower in children with urgent operations when compared to children with elective operations (Figure 4)(Mann-Whitney, both P=0.007).
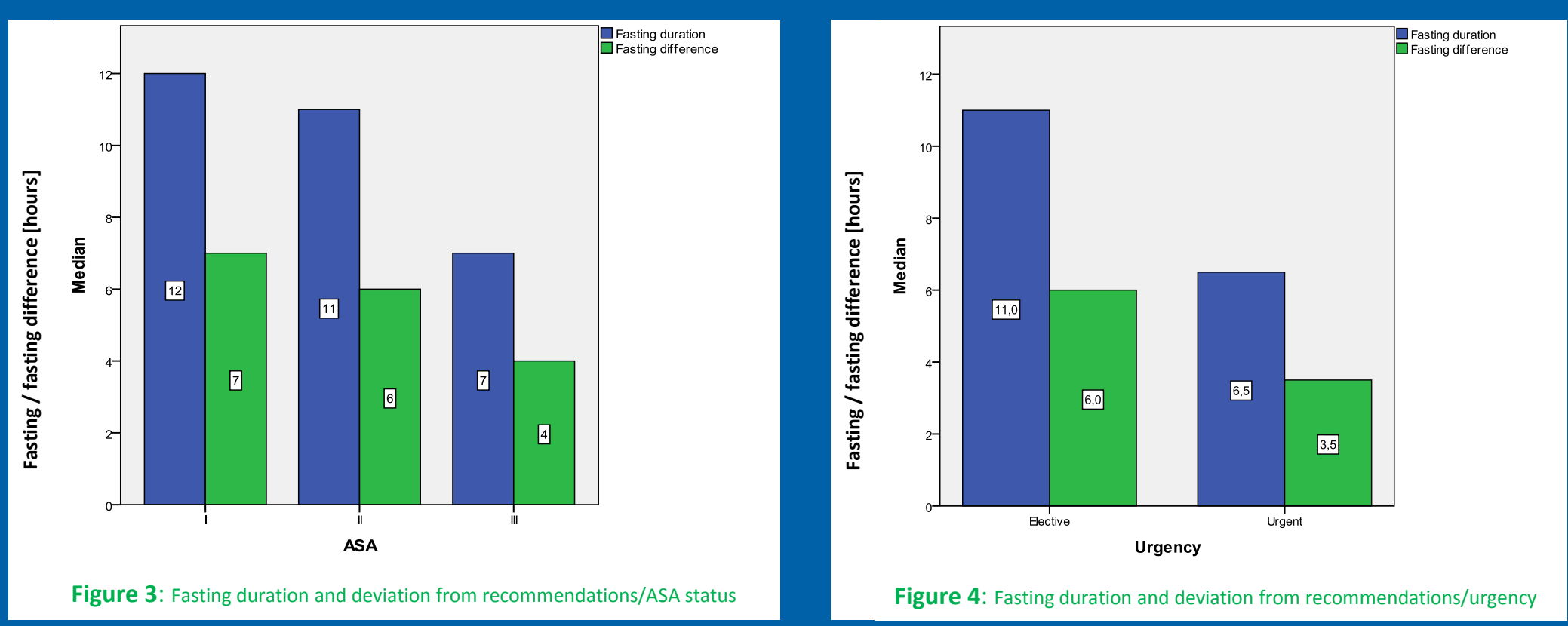

Conclusion

The prolonged fasting time in our hospital could be attributed to several factors. In order to reduce the deviations from fasting guidelines, anaesthesiologists need to improve their communication with the ward staff, 